\title{
Ultra-pure films and software
}

Mini-electron-beam evaporators and FDA-compliant X-ray microanalysis software

EGN1, EGN4, EGC04, H-PEB4

Mini-electron-beam evaporators

A series of four mini-

electron-beam

evaporators, the EGN1,

EGN4, EGC04 and

H-PEB4 are offered from

Oxford Applied Research.

These have the ability to

deposit ultra-pure films

of refractory materials,

from power sources in the

range $150-800 \mathrm{~W}$. Fully

controllable deposition

rates of between

$<0.1 \AA \mathrm{min}^{-1}$ and

$>300 \AA \mathrm{min}^{-1}$ are possible

for many materials. Co-

evaporation of up to four

materials is also possible

with models EGC04 and

H-PEB4, with

independent regulation

of the individual

deposition rates from a

E-SIG

EDAX

www.edax.com

X-ray microanalysis software

EDAX introduce E-SIG, which is claimed to be the first X-ray microanalysis software package that provides electronic signatures as required by 21 CFR (Code of Federal Regulations) part 11, and is able to satisfy US Food and Drugs Administration security and audit trail requirements. Spectra collected from the GENESIS software can be electronically archived along with user information and a date and time stamp. The three main features that ensure

single power supply. The evaporant - fitted either as a rod or contained within crucibles - is held at high voltage, which acts to draw electric current from a nearby hot filament, the impact of which causes evaporation. During the evaporation a small fraction of the vapour is ionized, which is harnessed to determine the deposition rate without the need for film thickness monitors during evaporation. Flux monitoring plates measure the ion current in the beam and extract most of the ions, leaving

data integrity are that the primary data cannot be altered; any changes to the data are tagged and tracked; and the levels of data processing are restricted on the basis of the relevant set permissions. For example, changes to parameters to improve peak

identification are saved, including the audit name and sequence number, to maintain audit trails. If data is printed out, that data is automatically saved with a new sequence number, if the parameters are different from those last saved.

These notes are compiled in the Nature Materials office from information provided by the manufacturers.

\section{www.oaresearch.com}

the beam predominantly neutral. Shutters are fitted as standard, and in EGC04 and H-PEB4 they allow several combinations of pockets to be selectively opened. Crucibles are suitable for use with insulators, materials that require

evaporation

temperature much higher than the melting point, or which have high

thermal conductivity.

\section{nature materials}

The 'New on the Market' section of Nature Materials is designed to give our readers access to the latest technologies, products and services available in the highly competitive materials science and engineering fields. With a worldwide circulation, Nature Materials is an invaluable resource for all scientists, in both academia and industry, who are active in the process of discovering and developing materials and materials-related concepts. We would like to invite you to send your Press Release Information about your new technologies, products and services for FREE inclusion in the feature section 'New on the Market'.

Weblinks will accompany each PR and some pictures will also be included. As an editorial page of Nature Materials, these PRs will also appear online.

For further information please contact Gerard Preston, Senior European Advertising Manager

By email:

g.preston@nature.com

By post:

Nature Publishing Group,

The Macmillan Building, Crinan Street, London, N1 9XW England.

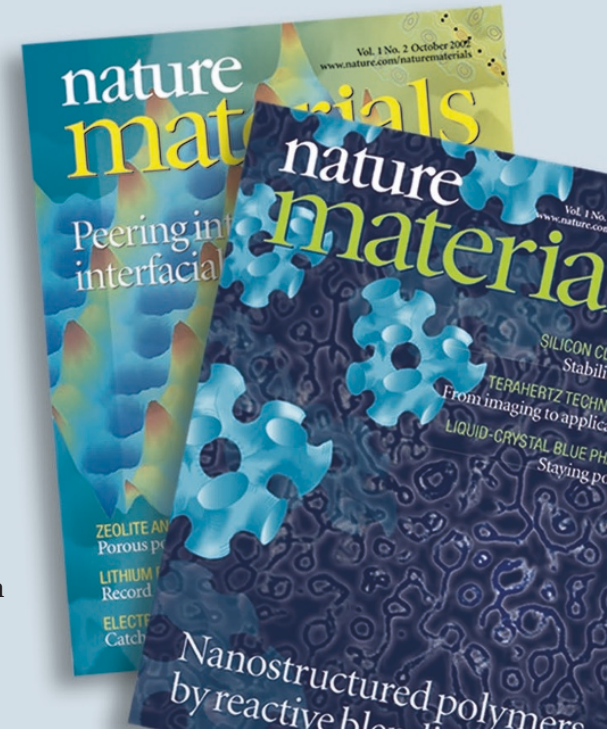

